\title{
An Observability Inequality On A Kind of Linear Parabolic Equation
}

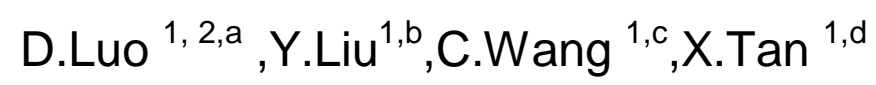

${ }^{1}$ The school of Mathematics of Zunyi Normal college,Zunyi, Guizhou Province,563002,P.R. China

2 The school of Mathematics and Statistics of Guizhou University, Guiyan, Guizhou Province, 550025,P.R. China

adsluo99@126.com, ${ }^{\text {b5 } 546692653 @ q q . c o m,, 2941359790 @ q q . c o m, ~}{ }^{\text {d } 2244761478 @ q q . c o m ~}$

Keywords: Nash inequality, Poincare inequality, semilinear parabolic equation, observability estimate

Abstract. In this paper, we study the observability inequality on a kind of linear parabolic equation. To show the observability estimate, we first derive the inequalities about the norms of the solution and its gradient, then reduce a lemma of inequality from a corollary directly[1]. At last, combing these inequalities with Nash inequality and Poincare inequality, we give the proof of the observability estimate.

\section{Introduction}

As we all know that observability estimate is a key in optimal control problem of linear partial differential equation, especial for the ones governed by linear parabolic equations. Observability can derive both exact controllability and approximate controllability directly or with some relevant conditions.Especially, in time optimal control problems, we often obtain null-controllability through observability estimate, to show the existence and bang-bang property of time optimal control problems[2-5].To the best of our knowledge, the observability estimates of parabolic equation system are main about the heat equation through Carleman inequality[6-8], or about semilinear parabolic equation with a discontinuous coefficient in one-dimension,or the case of coefficient with bounded variations of one-dimension, which proof relies on global Carleman estimate.But in the $n$-dimension case $(n \geq 2)$, the observability estimate of linear parabolic equation which coefficient has bounded variations is seldom talked about because the observability estimate dosen't completely rely on the corresponding Carleman inequality at all.

In this paper, consider the controlled system

$$
\begin{cases}y_{t}-\nabla \cdot[k(x) \nabla y]+a y=u \quad \text { in } \Omega \times(0,+\infty), \\ y=0 & \text { on } \partial \Omega \times(0,+\infty), \\ y(\cdot, 0)=y_{0} \quad \text { in } \Omega\end{cases}
$$

where $y_{0}$ is a function in $L^{2}(\Omega)$, and $u(\cdot, \cdot)$ is a control function take from the set of functions as follow:

$$
U \equiv\left\{u:[0,+\infty) \rightarrow L^{2}(\Omega) \quad \text { is measurable, }\|u(t)\|_{L^{2}(\Omega)} \leq M, \text { where } M \geq 0, \forall u\right\},
$$

where $0<\mu_{1}<k(x)<\mu_{2}$, a.e $x \in \Omega, \mu_{1}, \mu_{2}$ are positive numbers. $\forall T>0$ fixed, and it clear that the system(1) has a unique solution in $w[0, T]$, we denoted it as $y\left(\cdot ; y_{0}, u\right)$ or $y(x, t)$. To obtain the observability estimate, with the similar method in [9-10], we first derive the inequalities of the solution and the gradient $L^{2}(\Omega)$ norms, then reduce an inequality lemma about the initial and the final state from a corollary[1] directly. At last, combing the these inequalities with some other inequalities such as Nash inequality and Poincare inequality so on, we give the proof of the observability estimate. 


\section{The Observability Estimate of Linear Parabolic Equation}

In order to get the observability estimate, we consider the linear parabolic equation (2) corresponding to system(1.1). $\psi(x, t)$ denote the solution of

$$
\begin{cases}\psi_{\mathrm{t}}-\nabla \cdot(k(x) \nabla \psi)+a \psi=0 & \text { in } \Omega \times(0, T), \\ \psi(x, t)=0 & \text { on } \partial \Omega \times(0, T), \\ \psi(x, 0)=\psi_{0}(x) & \text { in } \Omega,\end{cases}
$$

where $\quad \psi_{0}(x) \in L^{2}(\Omega), \psi(x, t) \in C\left([0, T] ; L^{2}(\Omega)\right) \quad$ a.e $\quad x \in \Omega, a=a(x, t) \in L^{\infty}(\Omega \times(0, T)) \quad$ for convenience, we denote $\|a\|_{\infty}=\|a\|_{L^{\infty}(\Omega \times(0, T))}$.

Theorem2.1(observability estimate)Let $\Omega \times\left(t_{1}, t_{2}\right)$ be an open and non-empty subset of $\Omega$. Let $T>0$ and $E$ be a subset of positive measure in $(0, T)$. Then there is a constant $K(\Omega, \omega, E)$ such that for any

$$
\begin{gathered}
, \psi_{0} \in L^{2}(\Omega) \quad \text { the solution } \psi(x, t) \text { of (2.2) satisfies } \psi(x, t) \in C\left([0, T] ; L^{2}(\Omega)\right), \\
\|\psi(\cdot, T)\|_{L^{2}(\Omega)} \leq K(\Omega, \omega, E) \int_{\omega \times E}|\psi(x, t)| d x d t .
\end{gathered}
$$

Before proving the estimate theorem, we give three lemmas bellowing for proving the observability estimate, of which lemma2.1 is deduced from the corollary directly(see[1] corollary 1.4).

Lemma2.1. For each $T>0$ and $\psi_{0} \in L^{2}(\Omega)$, the solution $\psi(x, t)$ satisfies the estimate:

$$
\|\psi(x, T)\|_{L^{2}(\Omega)} \leq \sqrt{K_{1}}\left\|\psi_{0}(\cdot)\right\|_{L^{2}(\Omega)}^{\beta}\|\psi(\cdot, T)\|_{L^{2}(\omega)}^{1-\beta},
$$

where $\beta \in(0,1), K_{1}=e^{c\left(1+\frac{1}{\mu_{1} T}+T\left(\mu_{2}+\|a\|_{\infty}\right)\right)}$.

Lemma2.2. For any $\psi_{0} \in L^{2}(\Omega)$, the solution $\psi(x, t)$ of (2.1) satisfies the following two estimates: for any

$t \in(0, T]$

$$
\begin{aligned}
& \text { (i) } \int_{\Omega}|\psi(x, t)|^{2} d x \leq e^{2\left(\mu_{2}+\|a\|_{\infty}\right) t} \int_{\Omega}\left|\psi_{0}(x)\right|^{2} d x, \\
& \text { (ii) } \int_{\Omega}|\nabla \psi(x, t)|^{2} d x \leq \frac{c_{2} e^{2\left(\mu_{2}+\|a\|_{\infty}\right) t}}{t} \int_{\Omega}\left|\psi_{0}(x)\right|^{2} d x,
\end{aligned}
$$

where $K_{1}, c_{2}$ are constants and only depend on $n, \Omega$ with $\mu_{1} \leq k(x) \leq \mu_{2}, \forall x \in \Omega$.

Proof. (i).Multiplying the first equation of (2.1) by $\psi$,integrating it over $\Omega$, we get

$$
\frac{1 \mathrm{~d}}{d t} \int_{\Omega}|\psi|^{2} d x=-\int_{\Omega} k(\mathrm{x})|\nabla \psi|^{2} d x-\int_{\Omega} a \psi^{2} d x
$$

where $\psi=\psi(x, t)$, then by Poincare inequality we obtain

$$
\frac{d}{d t} \int_{\Omega}|\psi(x, t)|^{2} d x \leq 2 c_{1}\left(\mu_{2}+\|a\|_{\infty}\right) \int_{\Omega} \psi^{2} d x
$$

where $c_{1}=c_{1}\left(\mu_{2},\|a\|_{\infty}, \Omega\right)$ is a positive constant, and use Gronwall's inequality to get

$$
\int_{\Omega}|\psi(x, t)|^{2} d x \leq e^{2 c_{1}\left(\mu_{2}+|| a \|_{\infty}\right) t} \int_{\Omega}\left|\psi_{0}(x)\right|^{2} d x .
$$

(ii). Since $0<\mu_{1} \leq k(x) \leq \mu_{2}$ and $k(x) \in L^{\infty}(\Omega)$, from (i) we know that

$$
\frac{1 d}{2 d t} \int_{\Omega}|\psi(x, t)|^{2} d x+\mu_{1} \int_{\Omega}|\nabla \psi(x, t)|^{2} d x+\int_{\Omega} a|\psi(x, t)|^{2} d x=0,
$$

and with the result of (i), we obtain 


$$
\int_{\Omega}|\nabla \psi(x, t)|^{2} d x d t \leq \frac{c_{2} e^{2\left(\mu_{2}+\mid a \|_{\infty}\right) t}}{t} \int_{\Omega}\left|\psi_{0}(x)\right|^{2} d x
$$

where $c_{2}$ only depend on $\|a\|_{\infty}, \Omega, \mu_{1}$ and $T$.

Now we begin to present the proof of the observability estimate theorem, and the whole proof divides into two steps.

\section{Proof. Step 1}

For any $0 \leq t_{1} \leq t_{2} \leq T$, from lemma 2.1 and the Young inequality, $\forall \varepsilon>0$, we get

$$
\left\|\psi\left(\cdot, \mathrm{t}_{2}\right)\right\|_{L^{2}(\Omega)} \leq \varepsilon\left\|\psi\left(\cdot, t_{1}\right)\right\|_{L^{2}(\Omega)}^{\beta q}+\varepsilon^{\frac{-q}{p}} K_{1} e^{\frac{K_{2}}{t_{2}-t_{1}}}\|\psi(\cdot, t)\|_{L^{2}(\dot{\omega})}^{(1-\beta) q},
$$

where $p, q>1$ and $\frac{1}{p}+\frac{1}{q}=1, K_{1}=e^{\frac{c}{2(1-\beta)}\left(1+T\left(\|a\|_{\infty}+\mu_{2}\right)+\left(\mu_{2}+\|a\|_{\infty}\right)^{\frac{2}{3}}\right)}, K_{2}=\frac{c}{\mu_{1}(1-\beta)}, \omega \subset \omega \subset \Omega$.

Let $\beta p=1$, then $p=\frac{1}{\beta}$, since $\frac{1}{p}+\frac{1}{q}=1$, then

$$
\left\|\psi\left(\cdot, t_{2}\right)\right\|_{L^{2}(\Omega)} \leq \varepsilon\left(\left\|\psi\left(\cdot, t_{1}\right)\right\|_{L^{2}(\Omega)}\right)+\frac{1}{\varepsilon^{\frac{\beta}{1-\beta}}} K_{1} e^{\frac{K_{2}}{t_{2}-t_{1}}}\left\|\psi\left(\cdot, t_{2}\right)\right\|_{L^{2}(\omega)} \cdot
$$

Let $\alpha=\frac{\beta}{1-\beta}$, and by Nash inequality and Poincare inequality, $\forall \delta>0$ then

$$
\begin{gathered}
\left\|\psi\left(\cdot, \mathrm{t}_{2}\right)\right\|_{L^{2}(\omega)} \leq \\
\leq \psi\left(\cdot, \quad \mathrm{t}_{2}\right)\left\|_{L^{2}(\omega)}^{1+\frac{2}{n}}\right\| \psi\left(\cdot, \quad \mathrm{t}_{2}\right)\left\|_{L^{2}(\omega)}^{-\frac{2}{n}} \leq L\right\| \psi\left(\cdot, \mathrm{t}_{2}\right)\left\|_{L^{2}(\omega)}^{\frac{2}{n}}\right\| \nabla \psi\left(\cdot, \mathrm{t}_{2}\right)\left\|_{L^{2}(\omega)}\right\| \psi\left(\cdot, \mathrm{t}_{2}\right) \|_{L^{2}(\omega)}^{-\frac{2}{n}} \\
\leq \delta\left(\left\|\nabla \psi\left(\cdot, \mathrm{t}_{2}\right)\right\|_{L^{2}(\omega)}^{1-\frac{2}{n}}\right)^{p_{1}}+\delta^{-\frac{q_{1}}{p_{1}}}\left(E\left\|\psi\left(\cdot, \mathrm{t}_{2}\right)\right\|_{L^{1}(\omega)}^{\frac{2}{n}}\right)^{q_{1}},
\end{gathered}
$$

where $p_{1}, q_{1}>1, \frac{1}{p_{1}}+\frac{1}{q_{1}}=1, L$ and $L$ are constants only depending on $n, \Omega, \phi$. Let $\frac{(n-2) p_{1}}{n}=1$, Then

$$
\left\|\psi\left(\cdot, \mathrm{t}_{2}\right)\right\|_{L^{2}(\omega)} \leq \delta\left\|\nabla \psi\left(\cdot, \mathrm{t}_{2}\right)\right\|_{L^{2}(\omega)}^{1-\frac{2}{n}}+\delta^{-\frac{n-2}{2}} L^{\frac{n}{2}}\left\|\psi\left(\cdot, \mathrm{t}_{2}\right)\right\|_{L^{1}(\omega)}^{\frac{2}{n}}=\delta\left\|\nabla \psi\left(\cdot, \mathrm{t}_{2}\right)\right\|_{L^{2}(\omega)}^{1-\frac{2}{n}}+\frac{K_{3}}{\delta^{\frac{n-2}{2}}} E^{\frac{n}{2}}\left\|\psi\left(\cdot, \mathrm{t}_{2}\right)\right\|_{L^{1}(\omega)}^{\frac{2}{n}}
$$

where $K_{3}=E^{\frac{n}{2}}$.By lemma2.2, we know that

$$
\left\|\nabla \psi\left(\cdot, t_{2}\right)\right\|_{L^{2}(\Omega)} \leq \frac{K_{4}}{\sqrt{t_{2}-t_{1}}}\left\|\psi\left(\cdot, t_{1}\right)\right\|_{L^{2}(\Omega)},
$$

With $K_{4}=\sqrt{C_{2}} e^{\left(\mu_{2}+\|a\|_{\infty}\right) T}$.Combine the above three estimates, we gain

$$
\begin{aligned}
\left\|\psi\left(\cdot, t_{2}\right)\right\|_{L^{2}(\Omega)} \leq & \frac{K_{1} e^{\frac{K_{2}}{t_{2}-t_{1}}}}{\varepsilon^{\alpha}}\left\|\psi\left(\cdot, t_{2}\right)\right\|_{L^{2}(\omega)}+\varepsilon\left\|\psi\left(\cdot, t_{1}\right)\right\|_{L^{2}(\Omega)} \\
& \leq \frac{K_{1} e^{\frac{K_{2}}{t_{2}-t_{1}}}}{\varepsilon^{\alpha}} \delta \frac{K_{4}}{\sqrt{t_{2}-t_{1}}}\left\|\psi\left(\cdot, t_{1}\right)\right\|_{L^{2}(\Omega)}+\frac{K_{1} e^{\frac{K_{2}}{t_{2}-t_{1}}}}{\varepsilon^{\alpha}} \cdot \frac{K_{3}}{\delta^{\frac{n-2}{2}}}\left\|\psi\left(\cdot, t_{2}\right)\right\|_{L^{1}(\omega)}+\varepsilon\left\|\psi\left(\cdot, t_{1}\right)\right\|_{L^{2}(\Omega)} .
\end{aligned}
$$

Since $\varepsilon, \delta>0$ are arbitrary, let $\frac{K_{1} e^{\frac{K_{2}}{t_{2}-t_{1}}} K_{4}}{\varepsilon^{\alpha} \sqrt{t_{2}-t_{1}}} \delta=\varepsilon$, then 


$$
\left\|\psi\left(\cdot, t_{2}\right)\right\|_{L^{2}(\Omega)} \leq \frac{K_{1}^{\frac{n}{2}} K_{3} K_{4}^{\frac{n}{2}} e^{\frac{n K_{2}}{2\left(t_{2}-t_{1}\right)}}}{\left(t_{2}-t_{1}\right)^{\frac{n}{4}}} \cdot \frac{2^{\frac{n \alpha+n-2}{2}}}{(2 \varepsilon)^{\frac{n \alpha+n-2}{2}}}\left\|\psi\left(\cdot, t_{2}\right)\right\|_{L^{1}(\omega)}+2 \varepsilon\left\|\psi\left(\cdot, t_{1}\right)\right\|_{L^{2}(\Omega)} .
$$

Denote $\gamma=\frac{(\alpha+1) n-2}{2}, K_{5}=2^{\frac{(\alpha+1) n-2}{2}} K_{2}^{\frac{n}{2}} K_{3} K_{4}^{\frac{n-2}{2}} \cdot \frac{1}{\left(t_{2}-t_{1}\right)^{\frac{n-2}{4}}}, K_{6}=\frac{n K_{2}}{2}$, therefore

$$
\left\|\psi\left(\cdot, t_{2}\right)\right\|_{L^{2}(\Omega)} \leq \frac{K_{5} e^{\frac{K_{6}}{t_{2}-t_{1}}}}{(2 \varepsilon)^{\gamma}}\left\|\psi\left(\cdot, t_{2}\right)\right\|_{L^{1}(\omega)}+2 \varepsilon\left\|\psi\left(\cdot, t_{1}\right)\right\|_{L^{2}(\Omega)} .
$$

\section{Step 2.}

Let $E$ be a subset of positive measure in $(0, T)$, let $\ell$ be a density point of $E$, using proposition 2.1[9],for each $\tau>1$, there exists $\ell_{1} \in(\ell, T)$, depending on $\tau$ and $E$, such that the sequence $\left\{\ell_{m}\right\}_{m \geq 1}$, given by $\ell_{m+1}=\ell+\frac{1}{\tau^{m}}\left(\ell_{1}-\ell\right)$, satisfies

$$
\ell_{m}-\ell_{m+1} \leq 3\left|E \cap\left(\ell_{m+1}, \ell_{m}\right)\right| \text {. }
$$

Next, let $0<\ell_{m+2}<\ell_{m+1} \leq t<\ell_{m}<\ell_{1}<T$. We apply the above estimate to get

$$
\|\psi(\cdot, t)\|_{L^{2}(\Omega)} \leq \frac{K_{5} e^{\frac{n K_{6}}{2\left(t_{2}-t_{1}\right)}}}{\tilde{\varepsilon}^{\gamma}}\|\psi(\cdot, t)\|_{L^{1}(\omega)}+\tilde{\varepsilon}\left\|\psi\left(\cdot, t_{m+2}\right)\right\|_{L^{2}(\Omega)}, \forall \tilde{\varepsilon}=2 \varepsilon, \varepsilon>0 .
$$

Recall that by lemma 2.2.

$$
\begin{aligned}
\left\|\psi\left(\cdot, \ell_{m}\right)\right\|_{L^{2}(\Omega)} \leq & e^{\left(\mu_{2}+\|a\|_{\infty}\right)\left(\ell_{m}-t\right)}\|\psi(\cdot, t)\|_{L^{2}(\Omega)} \text {, therefore } \\
\left\|\psi\left(\cdot, \ell_{m}\right)\right\|_{L^{2}(\Omega)} & \leq e^{\left(\mu_{2}+\|a\|_{\infty}\right)\left(\ell_{m}-t\right)}\left(\frac{K_{5} e^{\frac{K_{6}}{t-\ell_{m+2}}}}{\tilde{e}^{\gamma}}\|\psi(\cdot, t)\|_{L^{1}(\omega)}+\tilde{\varepsilon}\left\|\psi\left(\cdot, \ell_{\mathrm{m}+2}\right)\right\|_{L^{2}(\Omega)}\right) \\
& \leq \frac{K_{7} e^{\frac{K_{6}}{t-\ell_{m+2}}}}{\varepsilon^{\gamma}}\|\psi(\cdot, t)\|_{L^{1}(\omega)}+\varepsilon\left\|\psi\left(\cdot, \ell_{m+2}\right)\right\|_{L^{2}(\Omega)},
\end{aligned}
$$

where let $\bar{\varepsilon}=e^{\left(\mu_{2}+\|a\|_{\infty}\right) T} \tilde{\varepsilon}$, and still signed as $\varepsilon$, and $K_{7}=K_{5}\left[e^{\left(\mu_{2}+\|a\|_{\infty}\right) T}\right]^{\gamma+1}$. Integrate it over

$$
\begin{aligned}
& E \cap\left(\ell_{m+1}, \ell_{m}\right) \text {, from } \ell_{m+1}=\ell+\frac{1}{\tau^{m}}\left(\ell_{1}-\ell\right) \text { and }(10) \text {, then } \\
& \qquad\left\|\psi\left(\cdot, \ell_{m}\right)\right\|_{L^{2}(\Omega)} \leq \frac{3}{\tau} \cdot \frac{K_{7} e^{\frac{2 K_{6} \tau^{m+1}}{\left(\ell_{1}-\ell\right)(\tau-1)}}}{\varepsilon^{\gamma} K_{6}} \int_{\ell_{m+1}}^{\ell_{m}} \chi_{E}\|\psi(\cdot, t)\|_{L^{1}(\omega)} d x+\varepsilon\left\|\psi\left(\cdot, \ell_{m+2}\right)\right\|_{L^{2}(\Omega)},
\end{aligned}
$$

take $d=2 K_{6} \frac{1}{\left(\ell_{1}-\ell\right) \tau(\tau-1)}$ and $\varepsilon=e^{-d \tau^{m+2}}$, then

$$
\mathrm{e}^{-d \tau^{m+2}(\gamma+1)}\left\|\psi\left(\cdot, \ell_{m}\right)\right\|_{L^{2}(\Omega)}-e^{-d \tau^{m+2}(\gamma+2)}\left\|\psi\left(\cdot, \ell_{m+2}\right)\right\|_{L^{2}(\Omega)} \leq K_{8} \int_{\ell_{m+1}}^{\ell} \chi_{E}\|\psi(\cdot, t)\|_{L^{1}(\omega)} d t,
$$

where $K_{8}=\frac{3 K_{7}}{\tau K_{6}}$, furthermore, to keep the exponent of $\tau$ in accordance with subscript $\ell$ of $\psi(\cdot, \ell)$,let

$$
\begin{aligned}
& d \tau^{2}(\gamma+1)=d(\gamma+2) \text {, then } \tau=\sqrt{\frac{\gamma+2}{\gamma+1}} \text {, and } \\
& \quad \mathrm{e}^{-d \tau^{m}(\gamma+2)}\left\|\psi\left(\cdot, \ell_{m}\right)\right\|_{L^{2}(\Omega)}-e^{-d \tau^{m+2}(\gamma+2)}\left\|\psi\left(\cdot, \ell_{m+2}\right)\right\|_{L^{2}(\Omega)} \leq K_{8} \int_{\ell_{m+1}}^{\ell} \chi_{E}\|\psi(\cdot, t)\|_{L^{1}(\omega)} d t
\end{aligned}
$$


Setting $m=2 m^{\prime}$, and summing the above from $m^{\prime}=1$ to infinity, then

$$
\begin{aligned}
& \mathrm{e}^{-d \tau^{2}(\gamma+2)}\left\|\psi\left(\cdot, \ell_{2}\right)\right\|_{L^{2}(\Omega)}-e^{-d \tau^{4}(\gamma+2)}\left\|\psi\left(\cdot, \ell_{4}\right)\right\|_{L^{2}(\Omega)}+e^{-d \tau^{4}(\gamma+2)}\left\|\psi\left(\cdot, \ell_{4}\right)\right\|_{L^{2}(\Omega)}-e^{-d \tau^{6}(\gamma+2)}\left\|\psi\left(\cdot, \ell_{6}\right)\right\|_{L^{2}(\Omega)}+\cdots \\
& +e^{-d \tau^{2 m^{\prime}}(\gamma+2)}\left\|\psi\left(\cdot, \ell_{2 m^{\prime}}\right)\right\|_{L^{2}(\Omega)}-e^{-d \tau^{2 m^{\prime}+2}(\gamma+2)}\left\|\psi\left(\cdot, \ell_{2 m^{\prime}+2}\right)\right\|_{L^{2}(\Omega)} \leq K_{8} \sum_{m^{\prime}=1}^{n} \int_{\ell_{2 m^{\prime}+1}}^{\ell} \chi_{E}\|\psi(x, t)\|_{L^{1}(\omega)} d t
\end{aligned}
$$

and

$$
\mathrm{e}^{-d \tau^{2}(\gamma+2)}\left\|\psi\left(\cdot, \ell_{2}\right)\right\|_{L^{2}(\Omega)} \leq K_{8} \int_{E}\|\psi(\cdot, t)\|_{L^{1}(\omega)} d t=K_{8} \int_{E \times \omega}|\psi(\cdot, t)| d x d t \text {, as } m^{\prime} \rightarrow \infty .
$$

Since $\|\psi(\cdot, T)\|_{L^{2}(\Omega)} \leq e^{2\left(\mu_{2}+\|a\|_{\infty}\right)\left(T-\ell_{2}\right)}\left\|\psi\left(\cdot, \ell_{2}\right)\right\|_{L^{2}(\Omega)}$, therefore

$$
\frac{\mathrm{e}^{-d \tau^{2}(\gamma+2)}\|\psi(\cdot, T)\|_{L^{2}(\Omega)}}{e^{2\left(\mu_{2}+\|a\|_{\infty}\right)\left(T-\ell_{2}\right)}} \leq \mathrm{e}^{-d \tau^{2}(\gamma+2)}\left\|\psi\left(\cdot, \ell_{2}\right)\right\|_{L^{2}(\Omega)} \leq K_{8} \int_{E \times \omega}|\psi(\cdot, t)| d x d t .
$$

Let $K(\Omega, \omega, E)=e^{2\left(\mu_{2}+\|a\|_{\infty}\right)\left(T-\ell_{2}\right)} K_{8}$, then

$$
\|\psi(\cdot, T)\|_{L^{2}(\Omega)} \leq K(\Omega, \omega, E) \int_{E \times \omega}|\psi(\cdot, t)| d x d t .
$$

The proof of observability estimate theorem is now completed.

\section{Acknowledgement}

In this paper, the research was supported by the National Nature Science Foundation of China (Grants no. 71461027, 71471158);Science and technology of Guizhou province outstandin g youth (Qian ke he ren zi [2015]06);Guizhou science and technology cooperation plan (Qian Ke He LH zi [2015]7004,[2016]7028,7031).

\section{References}

[1] Benabdallah A, Dermenjian Y, Le Rousseau J. On the controllability of linear parabolic equations with an arbitrary control location for stratified media[J]. Comptes Rendus Mathematique, 2007, 344(6): 357-362.

[2] V.Barbu,Analysis and Control of Nonlinear Infinite Dimensional Systems,Academic Press, Boston,1993.

[3] Udriste.Multitime Controllability, Observability and Bang-Bang Principle. Optim Theory Appl (2008)139: 141-157.

[4] Duyckaerts,E.,Zhang,X.,Zuazua,E.:On the optimality of the observability inequalities for parabolic and hyperbolic systems with potentials.Ann.Inst.H.Poincare Anal. Non Linaire 25,1- 41(2008).

[5] Benabdallah A, Dermenjian Y, Le Rousseau J. Carleman estimates for the one-dimensional heat equation with a discontinuous coefficient and applications to controllability and an inverse problem. Journal of Mathematical Analysis and applications,2007,336(2): 865-887.

[6] Le Rousseau J. Carleman estimates and controllability results for the one-dimensional heat equation with BV coefficients . Journal of Differential Equations, 2007, 233(2): 417-447.

[7] Rousseau J L, Robbiano L. Carleman estimate for elliptic operators with coefficients with jumps at an interface in arbitrary dimension and application to the null controllability of linear parabolic equations. Archive for rational mechanics and analysis, 2010, 195(3): 953-990.

[8] K.D.Phung and G.Wang, Quantitative unique continuation for the semilinear heat equation in a 
convex domain, J.Funct. Anal.,259(2010),1230-1247.

[9] K.D.Phung, L.J.Wang and C.Zhang, Bang-bang property for time optimal control of semilinear heat equation, Ann. Inst.H.Poincare Anal.Non Linear, 31(2014),477-499.

[10]K.Kunisch and L.J.Wang, Time optimal controls of the linear Fitzhugh-Nagumo equation with pointwise control constraints,J.Math.Anal.Appl.,395(2012),114-130. 